\title{
Substantiation of technological approaches to creation of a functional product based on creamy pumpkin composition
}

\author{
Irina Bibik ${ }^{1, *}$, Alexander Luchay ${ }^{1}$, and Sergey Dotsenko ${ }^{1}$ \\ ${ }^{1}$ Far Eastern State Agrarian University, 86, Politeknicheskaya Str., Blagoveschensk, Russia
}

\begin{abstract}
Scientific research and practice show that a certain part of the population of the Russian Federation has nutritional disorders. In particular, the deficiency in $\beta$-carotene is within $20 \%$, vitamin $\mathrm{C}-25 \%$, iron $-35 \%$, zinc $-35-45 \%$. The analysis found that on the basis of widespread raw materials of plant origin and, in particular, carotenecontaining, it is possible and advisable to create functional products containing a complex of vitamin and mineral substances with a synergistic (strengthening) effect. At the same time, the assumption was used about the possibility and feasibility of extracting $\beta$-carotene from pumpkin pulp by the fatty component of cream with a mass fraction of fat from $10 \%$ to $20 \%$. Taking into account this approach, a basic technological scheme for obtaining food systems enriched with $\beta$-carotene has been developed. On the basis of the cream and pumpkin composition obtained according to one of the options, the recipe for the dessert chocolate paste "Armeyskaya" was developed. The possibility and expediency of creating a mayonnaise product with a relatively high protein content, a rational ratio of polyunsaturated fatty acids, as well as significant concentrations of vitamins has been substantiated. Rheological dependences and consumer properties of a mayonnaise product with a given physiological effect have been established. It was found that it contains an antioxidant complex: $\beta$ carotene + vitamin $\mathrm{C}+\mathrm{Zn}$, which has a synergistic effect. It makes it possible to recommend the product as having the effect of maintaining the activity of the cardiovascular system in accordance with the Russian national standard GOST R-54059-2010.
\end{abstract}

\section{Introduction}

An analysis of numerous studies based on literature data over the past 20 years shows that the health problems of the population caused by inadequate nutrition, both around the world and in our country, continue to worry scientists and force them to focus their efforts on solving the problem of healthy nutrition.

At the same time, scientists note a deficiency of protein substances, an irrational ratio of fatty acids $\omega_{3}$ and $\omega_{6}$, as well as vitamins and micronutrients as the main reason for the lack of healthy nutrition. According to the Institute of Nutrition of the Russian Academy of

\footnotetext{
* Corresponding author: bibik7irina@mail.ru
} 
Sciences, the most important violation of the nutritional status of the population of the Russian Federation is excessive consumption of animal fats with a deficiency of polyunsaturated fatty acids, complete proteins, vitamins (C, group B, E, $\beta$-carotene, etc.), selenium, zinc, iron and other macro- and microelements $[1-5]$.

The authors of these works carried out certain studies in the framework of creating new functional products that allow designing nutritional status. So, the author of work [6] proposed to use drugs of a synthetic nature.

However, according to the authors of [7-12], this approach is not rational. At the same time, in our opinion, the existing resources of plant origin, containing biologically active substances, as well as essential nutrients, allows solving the problem of creating functional food products (FFP) using innovative methods of transforming raw materials [12].

As established on the basis of sources [10, 13-15], a rational way of using $\beta$-carotene is its extraction from various anatomical parts of carrots, sea buckthorn, brown algae, and pumpkin. At the same time, vitamins and minerals, which form the composition of certain complexes, are highly effective due to the presence of a synergistic effect [7].

Thus, the analysis and practice show that the creation of food products of a given composition and properties, provided with significant amounts of biologically active and mineral substances, as well as a balanced content of amino and fatty acids, is an important national economic problem that needs to be addressed.

The aim of the study is to substantiate and develop a recipe for dessert paste with a given physiological effect.

Research objectives:

1) substantiate the possibility and advisability of creating a dessert paste product based on a creamy pumpkin composition;

2) develop a basic technological scheme for obtaining a protein-vitamin dispersed system (PVDS), as well as an insoluble creamy pumpkin residue (ICPR);

3) develop a recipe for dessert pasta based on ICPR and give its comparative biochemical characteristics.

\section{Materials and Methods}

The working hypothesis is based on the assumption about the possibility and feasibility of extracting $\beta$-carotene from the pumpkin pulp by the fatty component of cream with a mass fraction of fat $\mathrm{MFF}=10-20 \%$.

Moreover, according to the data of the academician of the RAS N.N. Lipatov and his students [16], the amino acid balance of the protein in milk cream increases with an increase in the mass fraction of fat in them in comparison with those for skim milk.

At the same time, the data of E.N. Artemova, L.N. Stodolnik et al. [10, 14] showed that pumpkin pulp contains $\beta$-carotene from 40 to $70 \mathrm{mg} / 100 \mathrm{~g}$, and also a number of other biologically active substances.

Thus, the possibility and expediency of obtaining compositions by a certain transformation of this type of raw material and milk cream is quite obvious.

In this regard, a basic technological scheme for obtaining products based on a creamy pumpkin composition has been developed, which is shown in Fig. 1. 


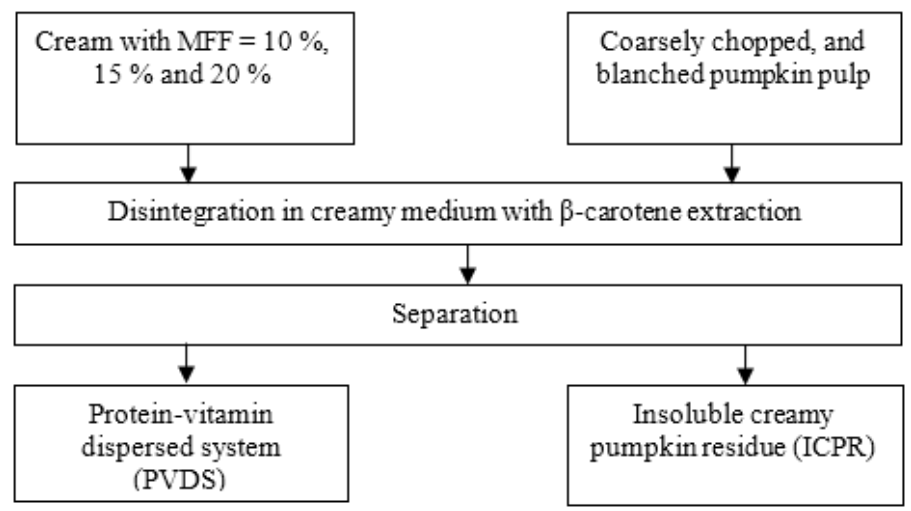

Fig. 1. Basic technological scheme for obtaining PVDS and ICPR.

According to the developed technological scheme, coarsely chopped and blanched pumpkin pulp of the Vitaminnaya variety is disintegrated in a creamy medium with MFF from $10 \%$ to $20 \%$ or more to an ultrafine and ultrahomogeneous state. Then, ICPR is separated from the obtained creamy pumpkin suspension, and a dessert paste is prepared on its basis. At the same time, the simultaneously obtained product in the form of PVDS can be used independently.

However, the program of further research suggests that we create on its basis an expanded range of functional products by, for example, thermoacid coagulation of proteins with a solution of ascorutin, vitamin PP, etc. with further transformation of the composition and properties of the resulting coagulum and whey (fortified).

Table 1 shows the recipe for the Armeyskaya dessert paste, and table 2 shows its comparative biochemical composition and energy value.

Table 1. Recipe for the Armeyskaya dessert paste.

\begin{tabular}{|l|c|}
\hline \multicolumn{1}{|c|}{ Components } & Mass fraction, \% \\
\hline ICPR & 40.0 \\
\hline Powdered milk & 10.0 \\
\hline Cocoa powder "Nutella" ${ }^{\circledR}$ & 50.0 \\
\hline
\end{tabular}

Table 2 shows comparative data on the biochemical composition of innovative dessert paste and chocolate paste "Nutella" ${ }^{\circledR}$.

\section{Results}

Analysis of the data in table 1 shows that in terms of the content of these substances, the innovative dessert paste meets the requirements for functional food products (FFP) with a given physiological effect defined by the national standard GOST R-54059-2010.

The developed method is protected by a patent for an invention [17].

According to the data obtained, the developed product can be attributed to the FFP with the code designation PFFI (physiologically functional food ingredients):

- vitamin C (V-I-1BE GOST R-54059-2010);

- carotenoids (V-II-B GOST R -54059-2010);

- zinc Zn (V-I-1 GOST R -54059-2010). 
Table 2. Comparative biochemical composition and energy value of dessert pastes ( $x \pm m ; p \leq 0.05)$.

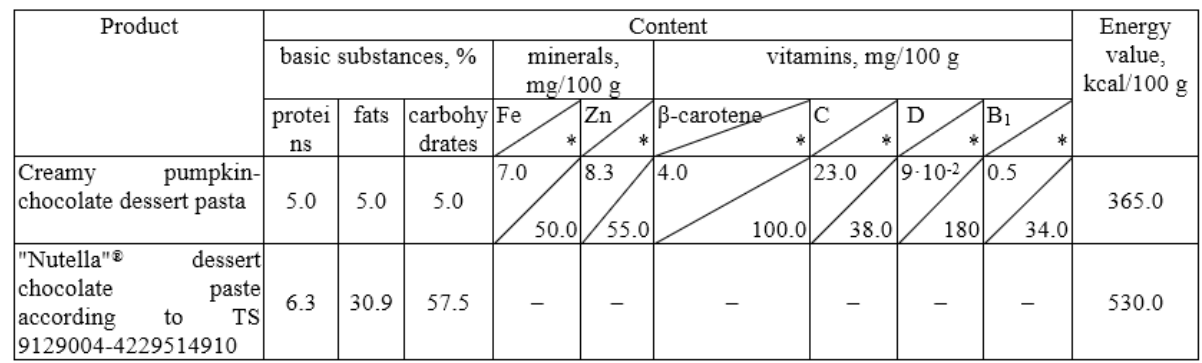

* $-\%$ from the daily norm of physiological need.

Continuing research in this area, a method for preparing a mayonnaise product of a functional orientation has been developed.

The development relates to the food industry and, in particular, to methods of preparing functional mayonnaise sauces.

There is a known method for preparing mayonnaise, including the joint homogenization of protein and lipid components, as well as other recipe components, in which coagulum is used as a protein component, and a blend of soybean and corn oils enriched with $\beta$-carotene extracted from carrots is used as a lipid component (Dotsenko S.M. et al. Technology of emulsion food products for specialized purposes // Food industry. 2014. No. 7. P. 37 - 41. Prototype).

The disadvantage of this method is the relatively low consumer properties due to the low content of $\beta$-carotene, with the simultaneous absence of flavonoids in the form of rutin, as well as relatively low organoleptic characteristics due to the absence of harmoniously combined creamy taste and pumpkin smell.

The object of the present invention is to obtain a functional mayonnaise product with relatively high consumer properties.

The technical result consists in the fact that this method allows obtaining a mayonnaise product with relatively high organoleptic characteristics in taste and smell with a given physiological effect of maintaining the cardiovascular system activity due to the presence of significant concentrations of $\beta$-carotene and the antioxidant complex "vitamin $\mathrm{C}+$ vitamin P".

This is achieved by the fact that a protein-lipid-vitamin coagulum is used as a protein component obtained by thermoacid coagulation of proteins in a soy-creamy-pumpkin dispersed system with a solution of ascorutin, and as a soy-corn-lipid component - a blend of oils with $\beta$-carotene extracted from pumpkin pulp of vitamin varieties, taken in the weight ratio coagulum : soybean-corn blend of oils $=1.5: 1.0$.

The method is carried out as follows.

At the beginning, a soybean-creamy-pumpkin dispersed system is prepared according to Fig. 2.

Then, on its basis, a protein-lipid-vitamin coagulum is prepared by thermoacid coagulation of proteins in it with an acid complex. The resulting coagulum is brought to the required moisture content by separating the whey product.

In parallel with this, a soy-corn blend is prepared with $\beta$-carotene extracted from the pulp of pumpkin.

The obtained coagulum containing proteins and lipids of plant and animal origin, fatsoluble vitamins $E$ and $\beta$-carotene, as well as vitamin $C$ and flavonoids in the form of rutin (vitamin $\mathrm{P}$ ) are mixed and homogenized to obtain a mayonnaise product.

Pre-prepared soybean seeds, for example the Sonata variety, containing significant concentrations of vitamin E, proteins and lipids, together with prepared pumpkin pulp of vitamin varieties, for example Vitaminnaya, in a weight ratio of 1: 1 are disintegrated, for 
example, in a buttermilk medium, obtaining a suspension, which is then divided into a soybean-buttermilk-pumpkin dispersed system (SBPDS) and an insoluble soybeanpumpkin residue.

In parallel with this, a creamy pumpkin dispersed suspension is prepared with subsequent separation of the insoluble creamy pumpkin residue from the protein-lipidvitamin dispersed system (PLVDS) containing significant concentrations of $\beta$-carotene.

The obtained SBPDS and PLVDS are mixed in a weight ratio of 1: 1 and homogenized. In the obtained protein-lipid-dispersed system, thermoacid coagulation is carried out with $5 \%$ buttermilk solution of ascorutin, obtaining a protein-lipid-vitamin coagulum (PLVC) enriched with fat-soluble vitamins $\mathrm{E}$ and $\beta$-carotene, as well as vitamins $\mathrm{C}$ and $\mathrm{P}$ with significant concentrations.

The obtained PLVC is mixed and homogenized with a lipid biocomplex based on a blend of soybean and corn oils enriched with $\beta$-carotene extracted according to the traditional scheme from the pulp of vitamin varieties of pumpkin in a weight ratio coagulum: blend $=1.5: 1$. Connection of two systems containing fat-soluble $\beta$-carotene provides its significant concentration in the resulting system by eliminating the so-called 'dilution' effect.

Table 3 shows the comparative biochemical composition of the proposed mayonnaise product, analogue and traditional "Provencal".

Analysis of the data in table 3 shows that with a rational daily intake of a mayonnaise product equal to 35 - 40 grams, a person's daily need for $\beta$-carotene will be satisfied only by $10.4 \%$, which indicates its insufficient concentration in the product. At the same time, the presence of a significant concentration of vitamin $\mathrm{P}$ in a daily dose of 40 grams, which is equal to $26.88 \%$, distinguishes the proposed mayonnaise from the prototype.

At the same time, in taste and smell (moderately pronounced creamy pumpkin), the proposed mayonnaise surpasses the prototype by 0.6 points, i.e. by $12.5 \%$.

Table 3. Comparative biochemical composition of mayonnaise products $(\overline{\mathrm{x}} \pm \mathrm{T} ; \mathrm{p} \leq 0.05)$.

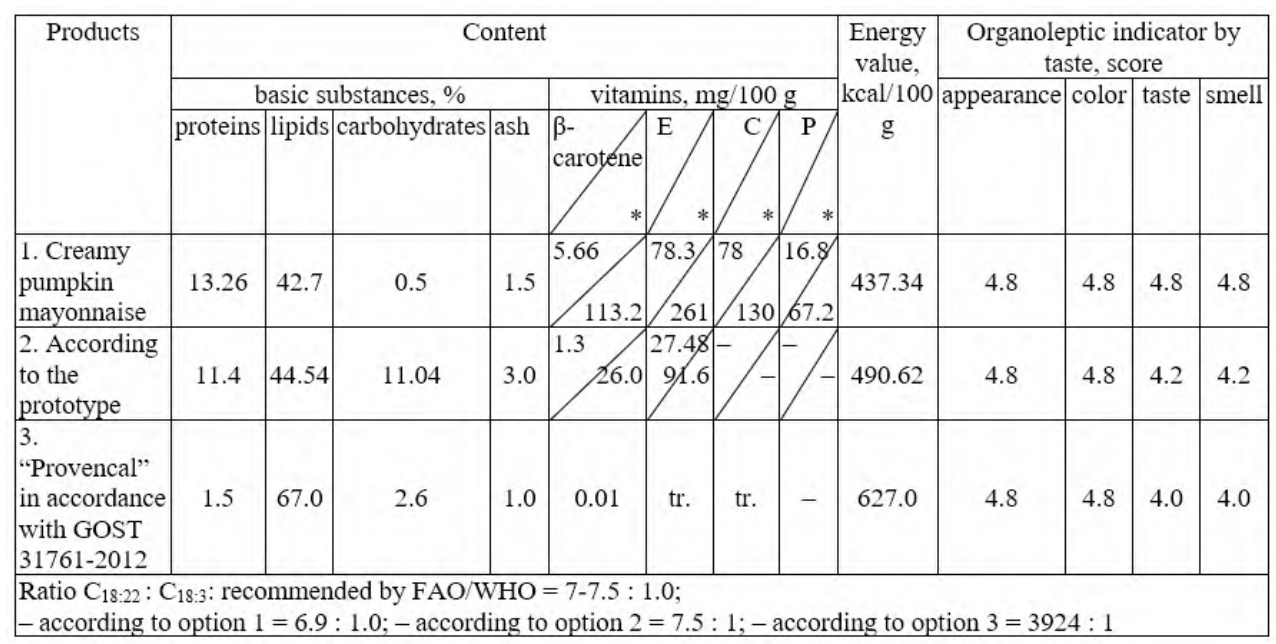

* _ \% from the daily norm of physiological need.

\section{Discussion}

Thus, the proposed mayonnaise product in its consumer properties meets the requirements for functional products, since has significant concentrations of $\beta$-carotene and flavonoids (vitamin P), as well as other vitamins (E and C). 
In this regard, it is functional with a given physiological effect of maintaining the activity of the cardiovascular system and has the following codes for physiologically functional ingredients in accordance with GOST R-52349-2005 and GOST R 54059-2010:

- vitamin C (V-I-1B GOST R-54059-2010);

- vitamin E (V-II-1;3B GOST R-54059-2010);

- $\beta$-carotene (V-II-1;3B GOST R-54059-2010)

- vitamin P (flavonoid) (V-I-4;5B GOST R-54059-2010);

- polyunsaturated fatty acids $\mathrm{C}_{18: 22}: \mathrm{C}_{18: 3}$ (V-I-2;3 GOST R-54059-2010).

A method for preparing a functional mayonnaise product includes mixing and homogenizing a protein, soybean-corn lipid, enriched with $\beta$-carotene components. A protein-lipid-vitamin coagulum obtained by thermoacid coagulation of proteins in a soycreamy-pumpkin dispersion system with a solution of ascorutin is used as a protein component, and a blend of oils with $\beta$-carotene extracted from vitamin pumpkin pulp is used as a soy-corn-lipid component enriched with $\beta$-carotene. They are taken in a following ratio by weight - coagulum: soybean-corn blend of oils - 1.5: 1.0.

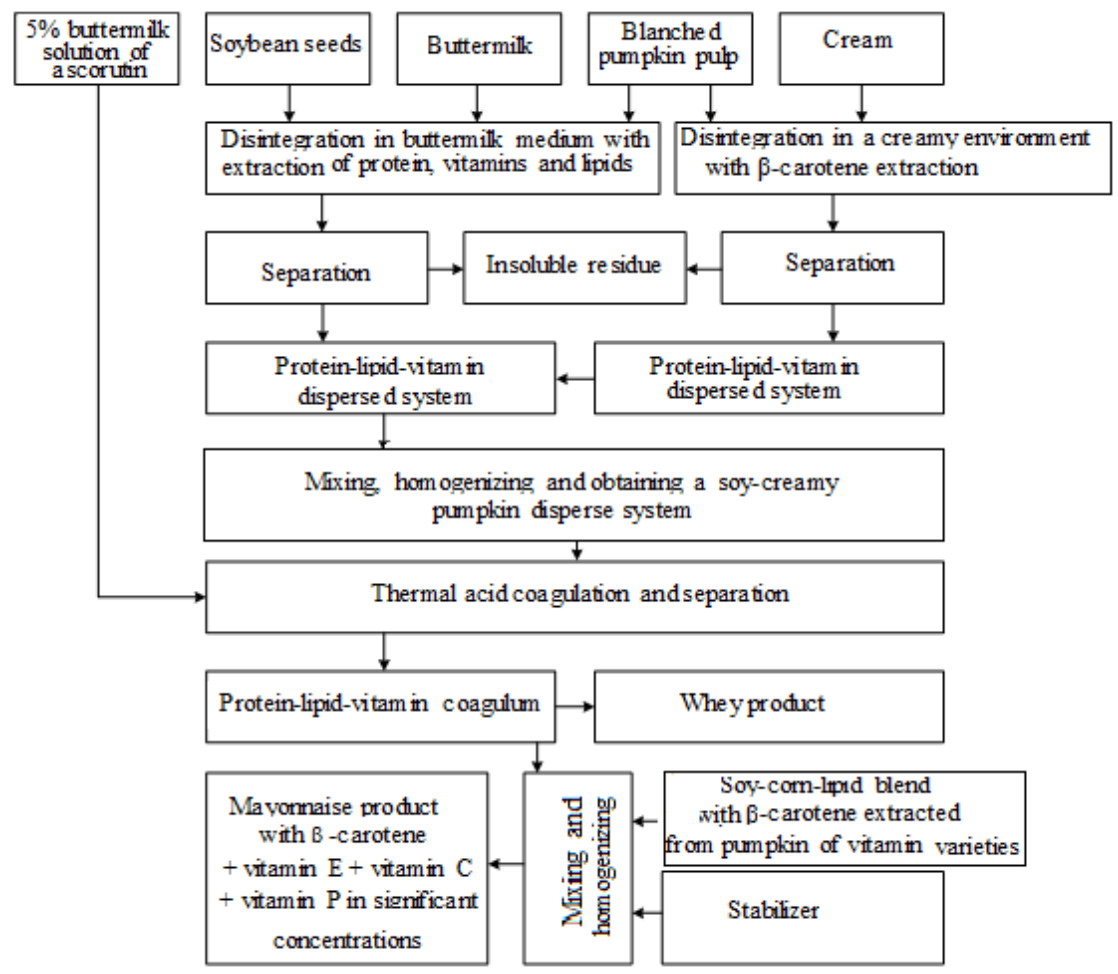

The developed mayonnaise has an attractive appearance, pronounced taste and aroma, rich orange-yellow color.

At the final stage of research, a dependence was determined that characterizes the change in effective viscosity $\eta$ on the storage time of the developed product $t_{\text {st. }}$ It is shown in Fig. 3. 


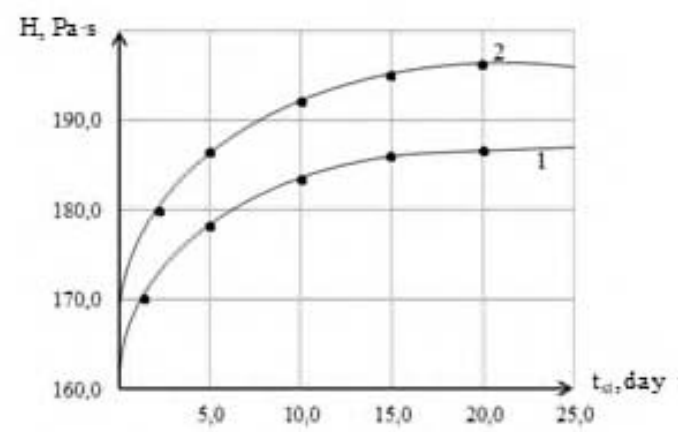

Fig. 3. Change in effective viscosity $\eta$ of mayonnaise product samples during storage 1 - soy-cream; 2 - control sample.

\section{Conclusion}

On the basis of the substantiated possibility and expediency of creating an FFP using a creamy pumpkin composition, a basic technological scheme for obtaining a dessert paste product "Armeyskiy" with an appropriate recipe has been developed.

Comparative biochemical composition of innovative and traditional products showed that the composition of the first one meets the requirements for FFP with a given physiological effect of maintaining the activity of the cardiovascular system.

The further research program provides for the creation of an expanded range of FFPs based on protein-vitamin-dispersed systems.

\section{References}

1. V.A. Tutelyan Nutrition and health Food industry, 5, 5 - 6 (2004)

2. A.A. Kudryashova The influence of nutrition on human health Food industry, 2. 67, .(2005)

3. A.F. Doronin, B.A. Shenderov Functional nutrition 295 (2002)

4. A.D. Durnev, L.A. Oganesyan, A.B. Lisitsyn Functional food products, Storage and processing of agricultural raw materials, 9, 15 - 21 (2007)

5. L.N. Shatnyuk, T.V. Spiricheva Scientific aspects of the use of innovative ingredients in the production of specialized food products Food ingredients. Raw materials and additives 2, 15-18 (2010)

6. N.L. Naumova. Scientific substantiation and practical aspects of the development of functional foods with antioxidant properties: abstract of thesis of doc. tech. sciences Chelyabinsk 34, (2017)

7. A.V. Shabrov, V.A. Dadali, V.G. Makarov. Biochemical bases of the action of food microcomponents, 189, (2003)

8. S.G. Kozlov.. Research and development of technologies for whey gel-like products using plant raw materials: abstract of thesis of doc. tech. sciences, 40 (2008)

9. L.V. Golubeva, E.I. Melnikova, E.B. Tereshkova Vegetable raw materials in milkcontaining dessert products, 2. 56 - 57 (2002)

10. E.N. Artemova, K.V. Daichenko The quality of emulsions based on flour from seeds of various pumpkin varieties Storage and processing of agricultural raw materials, 5, 65 67 (2009) 
11. N.N. Tipsina, E.A. Tipsin The use of carrot powder in the food industry Bulletin of KrasGAU 4, 257 - 261 (2014)

12. S.M. Dotsenko, I.V. Bibik. Scientific basis for the creation of functional food products using biologically active raw materials of the Far Eastern region Blagoveshchensk: Publishing House of Far Eastern State Agrarian University, 293 (2014)

13. E.T. Novitskaya Technology of forming the quality of emulsion nectars based on pumpkin (CUCURBITA MELON) with the addition of juice of honeysuckle berries, lemongrass, mountain ash, rose hips: abstract of thesis of Cand. tech. sciences, $\mathbf{2 4}$

14. L.N. Stodolnik. Substantiation and development of the technology of carotenecontaining products using brown algae for the production of flour confectionery and their commodity assessment: abstract of thesis of Cand. tech. sciences, 24 (2014)

15. V.I. Bobchenko Development of technology and commodity assessment of ice cream using processed plant materials containing functional ingredients 24 (2018)

16. N.N. Lipatov, T.Yu. Sazhinov, O.I. Bashkirov Formalized analysis of amino and fatty acid balance of raw materials, promising for the design of baby food with given food adequacy Storage and processing of agricultural raw materials. 8, $11-14$ (2001)

17. A.N. Luchay RF patent. Method for obtaining specialized products authors et al. Publ. in B.I. 6 dated 25.02.2019 